\title{
An Orientation-Independent Multi-Input Energy Harvesting Wireless Sensor Node
}

\author{
Kawsar Ali, Member, IEEE and Dan Rogers, Senior Member, IEEE
}

\begin{abstract}
Deploying energy-harvesting based wireless sensor nodes in challenging environments often means we do not have precise control over the placement and orientation of the nodes. This paper introduces a novel smart WSN whose operation does not depend on its placement and orientation. The proposed WSN unit is a cubeshaped device, each side of which is a complete wireless sensor module, sharing a common energy storage and a common communication bus with the other faces. An energy-efficient algorithm is designed based on the concepts of 'strongest energy face' and 'strongest radio face'. The proposed algorithm facilitates energy and information sharing among the faces of the cube with the common goal of harvesting the maximum possible energy from all sides, aggregating the energy on the shared energy bus, and then using the strongest radio face to transmit the sensed signal to the base station. The concept is verified in a $50 \mathrm{~cm}^{3}$ laboratory prototype built with off-the-shelf components. The experimental results show that the device successfully transmits the sensed data even in the worst case where only one face, which may not be the transmitting face, is harvesting energy.
\end{abstract}

Index Terms-energy harvesting, smart sensor, wireless sensor node (WSN)

\section{INTRODUCTION}

W IRELESS sensor nodes (WSNs) play an important role in today's emerging technologies such as the Internet of Things (IoT) and the Industrial Internet of Things (IIoT) that sense and collect data [1]. On-board harvesting of ambient energy has become an integral part of WSNs in order to increase their operating lifetime [2]-[5]. Typically such energy harvesting WSNs harvest energy from ambient sources like visible light, thermal gradient, vibration, radio frequency (RF) signals etc. The placement and orientation of such a WSN is extremely important [6] because, since energy input is directional, the yield of the energy harvester depends on its proper alignment with respect to the incident energy. For example, a photo-voltaic (PV) cell should face the source of light. Secondly, the sensor node has to be placed in such a way that the base station (receiver) falls in the line-of-sight of the antenna of the sensor node in order to ensure reliable radio communication. In addition, movement of the source of

Manuscript received August 07, 2019; revised November 26, 2019; accepted December 25, 2019. This work was supported by the Engineering and Physical Sciences Research Council (EPSRC), UK under grant number EP/P017436/1.

Kawsar Ali (corresponding author) and Dan Rogers are with the Department of Engineering Science, University of Oxford, United Kingdom (e-mail: kawsar.ali@eng.ox.ac.uk, dan.rogers@eng.ox.ac.uk). energy, the WSN, or the base station over time may lead to reduced energy harvest and loss of connectivity. For instance, the movement of the sun across the sky over a day affects the the energy harvest of a PV cell with a fixed orientation, or a base station placed on an aircraft will move moment-tomoment.

The sensitivity of energy harvesting to orientation can be resolved by adding extra energy harvesting cells on different sides of the node and then combining them (in series, parallel or series-parallel) to harvest as much energy as possible from all possible directions [7]. However, it is well known that in a series combination of cells the current capacity is limited by the weakest cell, and in a parallel combination, the terminal voltage is limited by the weakest cell. Moreover, the large voltage drop across the protection diode in series and parallel combinations make them impractical in lowvoltage low-power energy harvesting applications like WSNs. A series-parallel combination as used in [7] is a practical choice, but as will be shown in the next section, there are better-performing alternatives. The second issue with the lineof-sight for radio communication can be solved only by careful placing of the sensor node (e.g. keeping the antenna facing upwards).

While the directional nature of harvestable ambient energy and radio communication may be relatively trivial in most industrial monitoring and building automation systems, it poses a major challenge to the deployment of WSNs in extreme and inaccessible environments, for example an active volcano, or a nuclear waste disposal site [8], [9]. Significant cost ( e.g. the use of special-purpose robots or drones) is incurred to ensure proper placement and orientation of the WSNs in such cases.

To address the above-mentioned challenge, this paper introduces a WSN that does not require orientation. The unit is a cube-shaped device, each side of which is a complete energy harvesting wireless sensor module comprising an energy harvester (EH), a power management integrated circuit (PMIC), a microcontroller (MCU), a radio frequency (RF) transceiver (radio), and an array of sensors. All sides share a common energy storage unit and a common communication bus. Each side can work independently, but is also able to act co-operatively with the rest of the sides. As shown in the next section, keeping the faces independent increases the total energy harvest, and also presents the possibility of reducing power consumption in the RF transceiver with the use of a directional antenna. A detailed description of the system is presented in Section III.

An example use-case of the proposed WSN is to throw or 
drop (e.g. from a drone) it into an inaccessible area from a safe distance. The device will land in a random orientation in the unknown environment. It is probable that

1) One of the faces will have the strongest incident energy, called the 'strongest energy face';

2) One of the faces will have the clearest line-of-sight with the RF base station, and therefore, likely the best RF link, and so is called the 'strongest radio face'.

The strongest energy face may not be the strongest radio face, and these may also change over time. An energy-efficient algorithm is proposed to decide the strongest radio face based on the RSSI values of the RF transceivers [10], [11]. Once the decision has been made, the RF transceivers on all faces other than the strongest radio face are turned off. The PMICs on all the faces continue to harvest energy from all directions using a simple maximum power point tracking (MPPT) technique [12], [13], and all these energies are aggregated in the shared energy storage unit to continue the sensing and data transmission through the single RF transceiver on the strongest radio face. The proposed algorithm is described step-by-step in Section IV, which leads to some of the important design considerations discussed in Section V. Finally, the concept is experimentally validated with an example laboratory prototype in Section VI.

Therefore, the contributions of this paper are as follows:

1) A smart wireless sensor node whose operation does not depend on its placement and orientation in its target environment.

2) An energy-efficient algorithm to identify the strongest radio face and support it using the energy harvested from all directions. It is shown that the algorithm does not need any pre-defined master/slave face, they are decided on-thego based on their available energy.

\section{AdVANTAGE OF INDEPENDENT-FACE ENERGY HARVESTING WIRELESS SENSOR MODULES}

Let us take the example of a cube-shaped device with $1 \mathrm{~cm}$ dimension. There are six faces each with an area of $1 \mathrm{~cm}^{2}$ where the PV cells are mounted for harvesting solar energy from all directions.

\section{A. Organisation of PV Cells}

Fig. 1 shows different options for connecting the PV cells in this system. It is easy to see that the all-series and the all-parallel configurations using six $1 \mathrm{~cm}^{2}$ cells in Figs. 1(a) and 1(b) respectively are impractically inefficient because of the protection diodes. These protection diodes typically have a forward voltage drop of about $0.3 \mathrm{~V}$ which is close to $50 \%$ of the PV cell open-circuit voltage.

By connecting multiple smaller cells in series in each face, the effect of the protection diode can be overcome as shown by the third configuration in Fig. 1(c), where each face has six cells and one protection diode connected in series to form a string. The strings from all the six faces are connected in parallel and then controlled by a single PMIC. We shall compare the total harvest of this series-parallel configuration
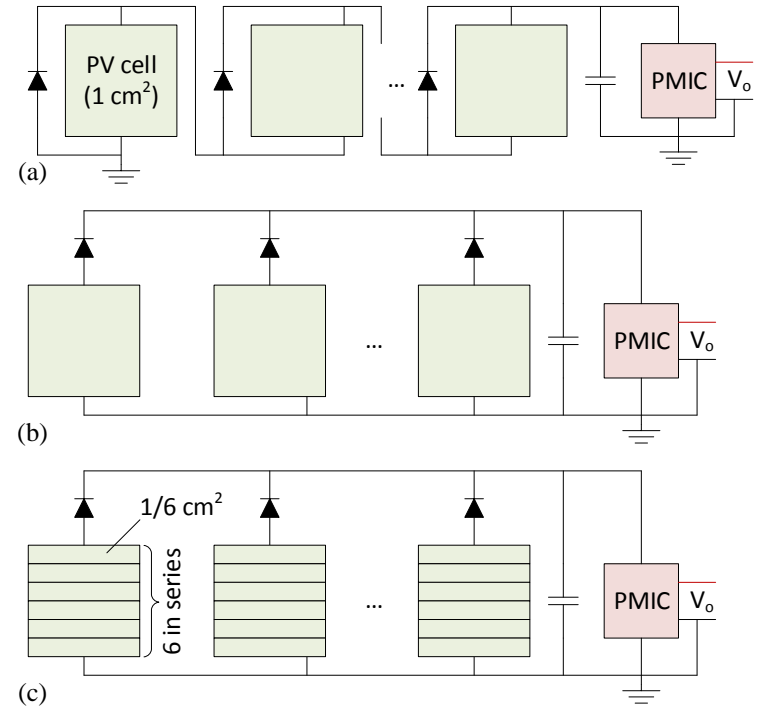

(c)

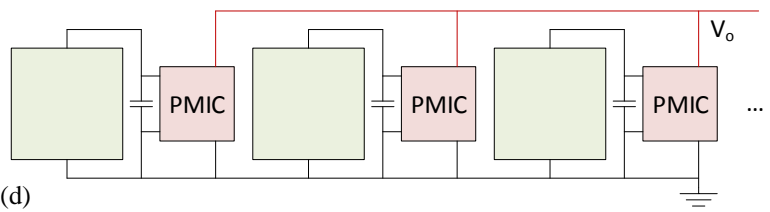

Fig. 1. Possible configurations of PV cells on a cube with dimension of $1 \mathrm{~cm}$ for harvesting solar energy from all directions: (a) all series, (b) all parallel, (c) series-parallel, and (d) independent (proposed).

with our proposed independent configuration where each face has a single PV cell of $1 \mathrm{~cm}^{2}$ area, and is interfaced by its individual PMIC as shown in Fig. 1(d). Note that the proposed configuration does not need any protection diode and the effective PV cell area is likely to be greater than that for the series-parallel configuration (Fig. 1(c)), because the area consumed by the cell borders and interconnection is reduced.

The output current $I$ of an ideal PV cell is a function of its terminal voltage $V$ and the relative solar irradiance $X$ (relative to 1 sun $=1000 \mathrm{~W} / \mathrm{m}^{2}$ ) as given by

$$
I(X, V)=X I_{\mathrm{SC}}-I_{0}\left(\exp \left(V / V_{\mathrm{TH}}\right)-1\right)
$$

where $I_{\mathrm{SC}}$ is the short-circuit current, $I_{0}$ is the reverse saturation current, and $V_{\mathrm{TH}}$ is the thermal voltage. Using (1), the total harvested power in the proposed independent configuration is given by

$$
P_{\text {proposed }}=\sum_{\mathrm{i}=1}^{6} V_{\mathrm{M}, \mathrm{i}} I_{\mathrm{i}}\left(X_{\mathrm{i}}, V_{\mathrm{M}, \mathrm{i}}\right)
$$

where $V_{\mathrm{M}, \mathrm{i}}$ is the terminal voltage of the PV cell on the $i$-th face at its maximum power point. This $V_{\mathrm{M}, \mathrm{i}}$ is set by individual PMICs on each face.

Similarly, the total harvested power for the series-parallel configuration in Fig. 1(c) is given by

$$
P_{\text {series-parallel }}=\sum_{\mathrm{i}=1}^{6} V_{\mathrm{COM}} I_{\mathrm{i}}\left(X_{\mathrm{i}}, V_{\mathrm{COM}}\right)
$$

where $V_{\mathrm{COM}}$ is the common terminal voltage set by the only PMIC, and it corresponds to the maximum power point of the 


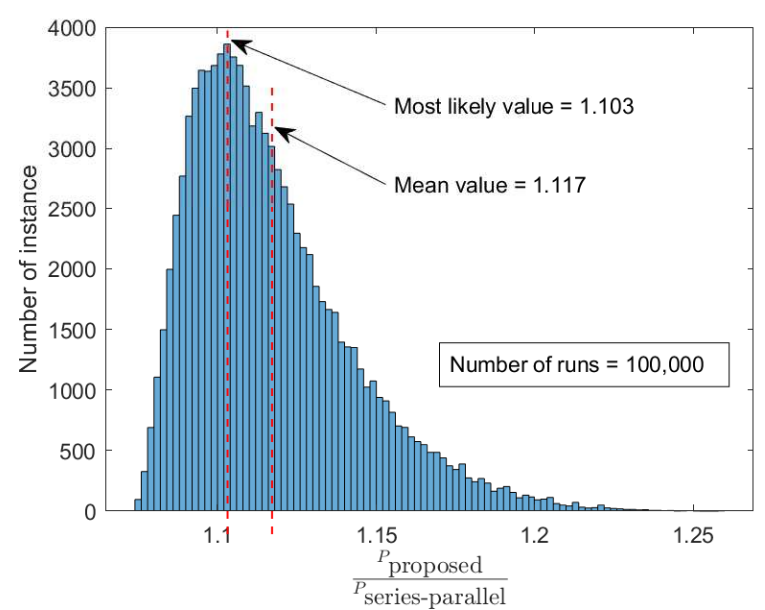

Fig. 2. Histogram of the ratio of total harvested power of the proposed independent configuration (Fig. 1(d)) to that of the series-parallel configuration (Fig. 1(c)), from Monte-Carlo simulation.

face receiving maximum irradiance. A clear disadvantage of this configuration is that no energy will be harvested from the face(s) having open-circuit voltage less than $V_{\mathrm{COM}}{ }^{1}$.

A Monte-Carlo simulation was run for the two cases (seriesparallel and proposed independent) using parameters of the solar cell AM-5610CAR from Panasonic with a uniform random distribution of the relative solar irradiance $X_{\mathrm{i}}$ such that $\sum X_{\mathrm{i}}=1$. Fig. 2 shows that there is a most-likely gain of $10.3 \%$ and an average gain of $11.7 \%$ in the total harvest for the proposed device.

\section{B. Multiple Directional RF Transceivers}

The other advantage of the proposed design is the possibility of using multiple directional antennas instead of a single omnidirectional antenna. The proposed device has six mutually orthogonal antennas arranged in a 3D space, and it will choose the best one among them in terms of link strength with the base station. Theoretically, the proposed device using a directional antenna could attain the same range using lesser radio power compared to the device using a single RF transceiver with a single omni-directional antenna. The exact reduction of radio power will depend on the design of the directional antenna, channel statistics, and alignment, among many other aspects, which are beyond the scope of this paper.

\section{SYSTEM DESCRIPTION}

A simplified schematic of the proposed system is shown in Fig. 3. It consists of six identical subsystems sharing a common energy storage bus and a communication bus. For ease of discussion, each subsystem is referred to as a 'face'

${ }^{1}$ This is because PMICs such as [14]-[17] perform simple MPPT by setting the terminal voltage to a fixed fraction of the open circuit voltage (often about $80 \%$ for PV aplications) as this is where the maximum power point is approximately located. It should be noted that the solar dice [7] from Texas Instruments, although a good example of the case in Fig. 1(c), does not perform any type of MPPT: it uses a simple buck converter as its PMIC which sets $V_{\mathrm{COM}}$ based on whether the output voltage has reached the desired value or not.

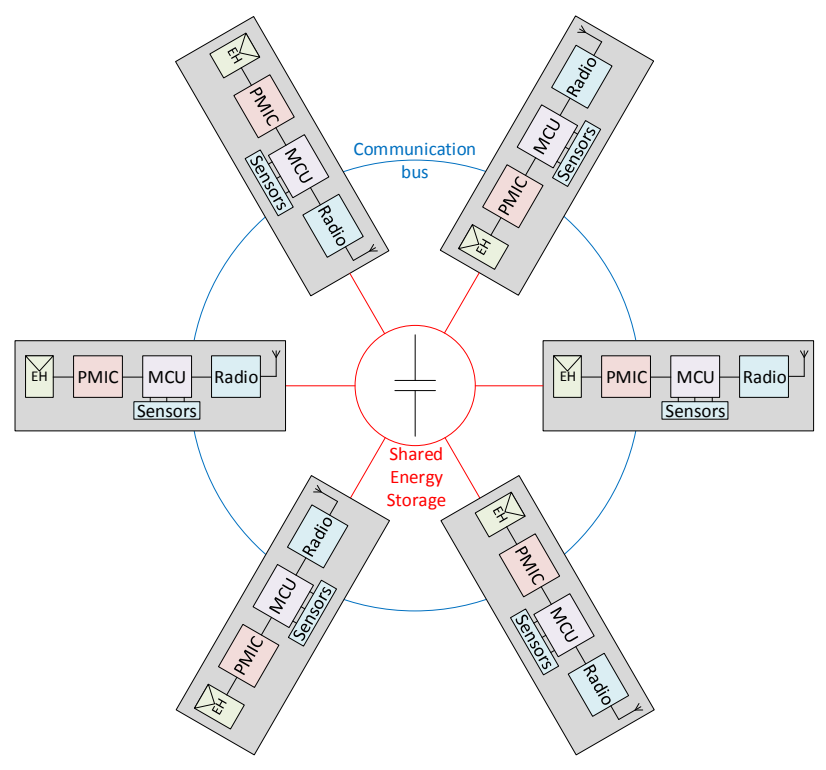

Fig. 3. Simplified schematic of the proposed system (cube). Each subsystem is a 'face' of the cube.

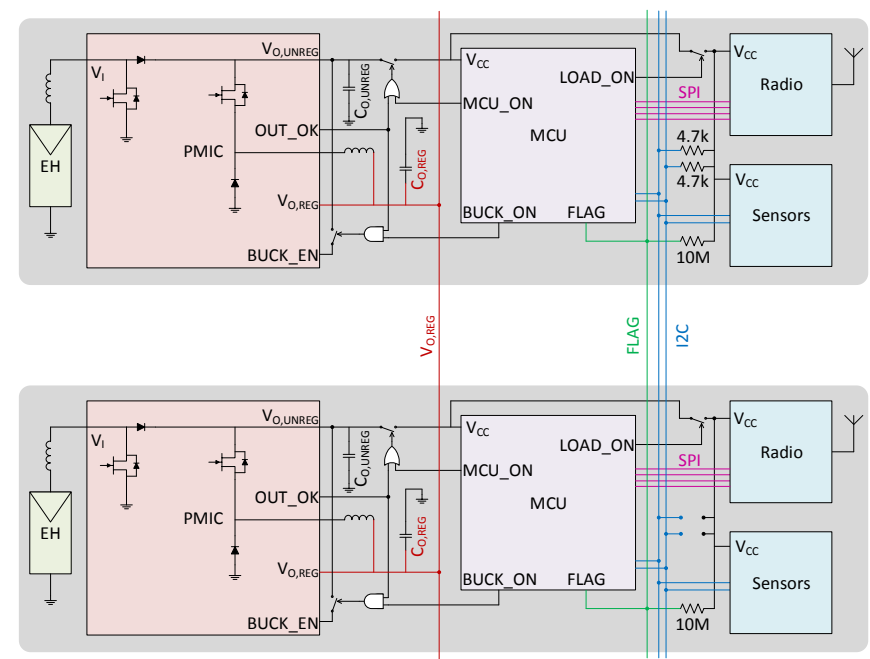

Fig. 4. Functional block diagram of the faces of the cube and illustration of the interconnection between two faces.

of the cube henceforth. As can be seen from Fig. 4, each face is an independent wireless sensor module comprising an energy harvester (EH), a power management integrated circuit (PMIC), necessary sensors, a microcontroller unit (MCU) and an RF transceiver (radio). In highly customized designs, the MCU and the radio are often integrated in a single systemon-chip (SoC) [18].

The PMIC is usually a charge pump based (e.g. LTC3108) [19]-[21] or an inductive switching (buck/boost) based (e.g. bq25570, LTC3105, SPV1050) [14]-[17], [22] power electronic converter that steps up or down the harvester voltage as required by the system load. These converters are highly optimized for very low voltage and very low power operation. Many of them also feature some basic MPPT (e.g. bq25570, LTC3105, SPV1050) [14]-[17] to manage the high output 
impedance of most EHs. The output port of the PMIC is usually unregulated $\left(V_{\mathrm{O} \text {,UNREG }}\right)$ with just an upper bound on the output voltage (e.g. bq25570, LTC3105). A hysteresiscontrolled output voltage GOOD or OK indicator (OUT_OK) is also provided (e.g. bq25570, LTC3105) [14]-[16], [19], which toggles high when $V_{\mathrm{O} \text {,UNREG }}$ is greater than $V_{\mathrm{OKH}}$, and toggles low when $V_{\mathrm{O}, \text { UNREG }}$ is less than $V_{\mathrm{OKL}}$. The OUT_OK signal is commonly used by an external load switch to turn on/off the system load. The voltage levels $V_{\mathrm{OKH}}$ and $V_{\mathrm{OKL}}$ are set by external resistive dividers. To cater to the need of a constant dc voltage in the system (e.g. to power the sensors for accurate measurement), most of the commercially available PMICs also offer a regulated output port $\left(V_{\mathrm{O}, \mathrm{REG}}\right)$ implemented by either a low drop out (LDO) regulator (e.g. LTC3105, SPV1050) [16], [17], [19] or a buck regulator (e.g. bq25570) [15]. This regulator can be enabled or disabled externally (using BUCK_EN as shown in Fig. 4) as and when it is needed. In the proposed system, the unregulated output ports are kept local to the respective faces, and the regulated ports are shared among all the faces to form a common energy storage bus.

The microcontroller implements the proposed algorithm (discussed in the next section), controls the radio, reads from the sensors, and also assists the PMIC in the overall power management of the system. Most of the microcontrollers used in WSN applications feature low power modes (LPM) [23], [24], where the central processing unit (CPU) and the individual peripherals can be turned-off selectively to minimize energy consumption when the system is sleeping. Note that the communication protocols (e.g. SPI and I2C) shown in Fig. 4 are example cases specific to this study. In practice, one is free to choose any suitable protocol. The roles of the general purpose input/output (GPIO) pins shown in Fig. 4 are as follows.

1) MCU_ON: is OR-ed with OUT_OK to keep the MCU on when $V_{\mathrm{OKL}}>V_{\mathrm{O}, \mathrm{UNREG}}>V_{\mathrm{CC}, \mathrm{MIN}}$. Here, $V_{\mathrm{CC}, \mathrm{MIN}}$ is the minimum supply voltage required for the MCU, which is typically $1.8 \mathrm{~V}$ for the MCUs used in WSN applications. The MCU can be turned off only when both OUT_OK and MCU_ON goes low.

2) BUCK_ON: is AND-ed with OUT_OK to ensure that the Buck regulator is disabled when $V_{\mathrm{OKL}}>V_{\mathrm{O}, \mathrm{UNREG}}>$ $V_{\mathrm{CC}, \mathrm{MIN}}$, even if the MCU is sleeping with BUCK_ON kept high. This prevents the sudden turn-off of the MCU during the initial charging phases of the shared output bus $\left(V_{\mathrm{O}, \mathrm{REG}}\right)$.

3) LOAD_ON: is used for turning on/off the load (i.e. the radio and the sensors).

4) FLAG: is externally pulled-up to $V_{\mathrm{CC}}$ with a high value resistor $(10 \mathrm{M} \Omega)$. This pin is polled by the MCU to determine whether it is the I2C master or the I2C slave.

To highlight the significance of the logic OR and the logic AND gates shown in Fig. 4, two other possible interconnections of the PMIC and the MCU are shown in Fig. 5. For the case in Fig. 5(a), when the buck converter is turned on by the MCU for the first time, the voltage $V_{\mathrm{O} \text {,UNREG may }}$ quickly fall below $V_{\mathrm{OKL}}$. This is because the output of the buck

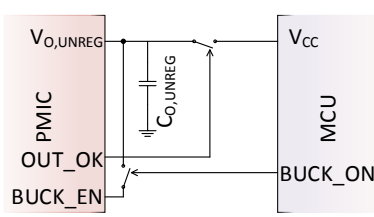

(a)

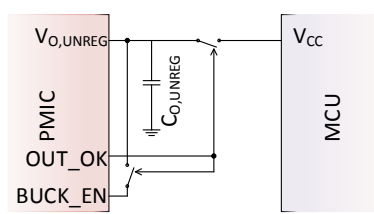

(b)
Fig. 5. Other possible but less energy-efficient interconnections of the PMIC and the MCU for the proposed application.

converter (the shared output bus $V_{\mathrm{O}, \mathrm{REG}}$ ), which is initially at zero voltage, has a relatively large capacitance $C_{\mathrm{O}, \mathrm{REG}}$, and energy will be rapidly drained into it. This will turn off the MCU, and hence, the buck converter as well. Such on-off cycling of the MCU will repeat until the shared output bus is charged to $V_{\mathrm{OKL}}$. Typically, the start-up sequence of the MCU consumes a lot of charge, and so a lot of energy will be wasted in these multiple on-off cycles. It is easy to see that a similar phenomenon will occur for the case in Fig. 5(b).

The configuration using the logic OR and the logic AND gates shown in Fig. 4 solves the above issue. Here, the OUT_OK signal is OR-ed with the MCU_ON signal from the MCU. When OUT_OK goes high at $V_{\mathrm{O}, \mathrm{UNREG}}=V_{\mathrm{OKH}}$, the MCU is turned on, and the first action of the MCU is to set MCU_ON high. After this, the MCU can be turned off only when both OUT_OK and MCU_ON goes low, which is not until $V_{\mathrm{O}, \mathrm{UNREG}}$ falls below $V_{\mathrm{CC}, \mathrm{MIN}}$. The AND gate on the buck control prevents the voltage $V_{\mathrm{O}, \mathrm{UNREG}}$ from falling below $V_{\mathrm{OKL}}$ (which is higher than $V_{\mathrm{CC}, \mathrm{MIN}}$ as shown in Fig. 6 ) by turning off the buck converter at $V_{\mathrm{O}, \mathrm{UNREG}} \leqslant V_{\mathrm{OKL}}$. The proposed configuration therefore avoids the multiple on-off cycles of the MCU, and is more energy efficient. The MCU, after being turned on, can go to sleep in its low power mode (LPM) keeping both MCU_ON and BUCK_ON high, and wait until the shared output bus is charged to the desired reference voltage $\left(V_{\mathrm{O}, \mathrm{REG}}^{*}=3.5 \mathrm{~V}\right.$ in this case $)$.

Note that all the pull-up resistors $(4.7 \mathrm{k} \Omega$ for $\mathrm{I} 2 \mathrm{C}$ and 10 $\mathrm{M} \Omega$ for FLAG in Fig. 4) are pulled-up using the MCU's input power supply $\left(V_{\mathrm{CC}}\right)$ after the LOAD_ON switch, and not using the shared output bus $\left(V_{\mathrm{O}, \mathrm{REG}}\right)$. This is done to prevent the large amount of leakage current through these pull-up resistors while $V_{\mathrm{O}, \mathrm{REG}}$ is building up and some of the MCUs are still not on, their IO pins possibly being in the 0 or GND state and not in the high impedance state. Also, unlike the FLAG pull-up resistors, the $\mathrm{I} 2 \mathrm{C}$ pull-up resistors are pulled-up from only one face to prevent back-powering the radios on the faces that are not awake yet.

\section{Proposed Algorithm}

This section explains the proposed algorithm. The goal of the algorithm is to find the strongest radio face based on the RSSI values of the radios. An I2C communication protocol among the MCUs with no pre-determined master/slave is used to achieve this. The MCU on the strongest energy face is expected to declare itself as the I2C master by pulling a FLAG high, and the other MCUs will become I2C slaves on reading 


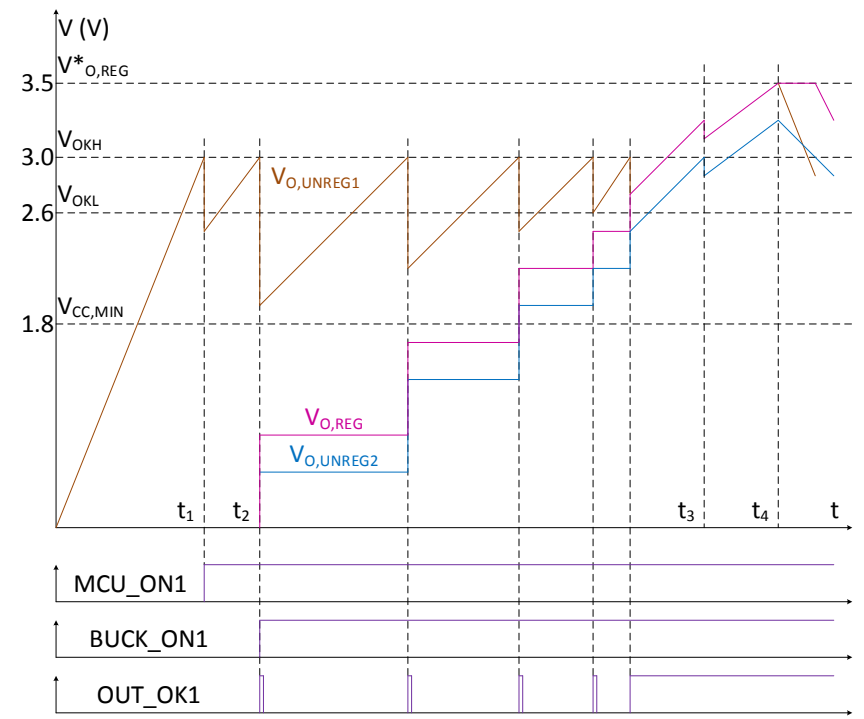

Fig. 6. Illustration of voltage build-up in the local and shared output buses using two faces. Note that the time axis is not to scale.

the FLAG high. Therefore, no particular MCU is assigned as master or slave permanently. All the MCUs are loaded (flashed) with the same program (code), and depending on whether an MCU becomes master or slave, the MCU executes the relevant part corresponding to master or slave behaviour. A unique address must be assigned to each MCU, however.

Fig. 6 shows an illustration of the voltage build-up in the local and shared output buses using the algorithm for an example case of two faces working together, only one of them having energy available at its input. The numeric values of different voltage levels are used for easy understanding. Note that these values are specific to this study, but the algorithm can be adopted to any other similar application as long as the following relation is satisfied (see Fig. 6).

$$
V_{\mathrm{O}, \mathrm{REG}}^{*}>V_{\mathrm{OKH}}>V_{\mathrm{OKL}}>V_{\mathrm{CC}, \mathrm{MIN}}
$$

Similarly, this is true for the sleep/wait times used here: the designer needs to choose these values based on the available average energy. The major steps of the algorithm are described below.

1) When the OUT_OK signal goes high for the first time (at $V_{\mathrm{O}, \mathrm{UNREG}}=V_{\mathrm{OKH}}=3 \mathrm{~V}$ at $t_{1}$ in Fig. 6), the MCU is turned on, and it sets its MCU_ON signal high.

2) The MCU then goes to sleep for a certain period (e.g. 1s in our case). The sleep period is essentially a low power mode (LPM) of the MCU using a low frequency auxiliary clock timer to periodically wake up from sleep. After waking up, the MCU checks if $V_{\mathrm{CC}}>V_{\mathrm{OKH}}$. If not, it goes back to sleep again. This keeps on repeating until $V_{\mathrm{CC}}>V_{\mathrm{OKH}}$, when the BUCK_ON signal is set high (at $t_{2}$ ). Note that $V_{\mathrm{CC}}$ is same as $V_{\mathrm{O}, \mathrm{UNREG}}$ after the turn-on of the MCU.

3) The MCU again enters a looping sleep cycle, sensing $V_{\mathrm{CC}}$ at every wake-up. The condition to exit from this loop is either $V_{\mathrm{CC}}>V_{\mathrm{O}, \mathrm{REG}}^{*}$ or if the FLAG is high. During this loop, the buck converter charges up the shared output bus in a stepped fashion as shown in Fig. 6 from $t_{2}$ to $t_{4}$. Towards the end of this step, the shared output bus voltage $V_{\text {O,REG }}$ becomes high enough to back-charge the local output ports $\left(V_{\mathrm{O}, \mathrm{UNREG}}\right)$ of all the weaker energy faces to a voltage level above $V_{\mathrm{OKH}}(=3 \mathrm{~V})$, and turn on their MCUs. The backcharging is possible because of the body-diode of the buck MOSFET (e.g. face2 MCU is turned on at $t_{3}$ in Fig. 6).

4) Clearly, the MCU on the strongest energy face will be the first one to satisfy the condition $V_{\mathrm{CC}}>V_{\mathrm{O}, \mathrm{REG}}^{*}$ and exit the sleep loop. After exiting, it declares itself as the I2C master and sets its LOAD_ON signal high, thereby pulling the FLAG high. Consequently, the MCUs on the other faces will also exit the sleep loop on reading the FLAG high and therefore, initialise themselves as I2C slaves. At this moment, all the MCUs set their LOAD_ON signals high which turns on their respective radios and sensors (at $t_{4}$ ).

5) After waking up, the radios try to JOIN any existing network and LINK to the base station. In the process the RSSI value of each radio gets updated, which is used as an indicator of its link strength. A random delay (0 to $1 \mathrm{~s}$ ) is added before the JOIN stage to facilitate clear channel assessment (CCA) in the radios.

6) Now the I2C master (strongest energy face) reads the RSSI values from all the other $\mathrm{I} 2 \mathrm{C}$ slave faces, decides the strongest radio face based on the maximum RSSI value, and writes the address of the strongest radio face to the other I2C slave faces.

7) Finally, based on the decision, each MCU decides to continue in one of the following ways. If it is the strongest radio face, it continues to periodically sense and transmit over radio to the base station. If it is not the strongest radio face, it puts its LOAD_ON signal low and goes to sleep keeping its MCU_ON and BUCK_ON signals high.

Thus, at the end of the algorithm, only the strongest radio face is left running. This face's radio transmission is supported cumulatively by the energy harvested by all the faces from all directions. Fig. 7 shows the flowchart of the proposed algorithm. Some important aspects to be noted are as follows:

1) Steps 1 to 3 will work in the same way for both cases of turning on of the MCU - either by its local bus $V_{\text {O,UNREG, }}$ or assisted by the shared bus $V_{\mathrm{O}, \mathrm{REG}}$.

2) The reference voltage of the shared output bus $V_{\mathrm{O}, \mathrm{REG}}^{*}$ should be set at least one diode-voltage-drop above $V_{\mathrm{OKH}}$ to ensure that the face with no available energy at its input can be woken up by the shared output bus. For example, in Fig. $6, V_{\mathrm{OKH}}=3 \mathrm{~V}$; therefore set $V_{\mathrm{O}, \mathrm{REG}}^{*}=3.5 \mathrm{~V}$ assuming the voltage drop across the body diode of the buck MOSFET is about $0.3 \mathrm{~V}$.

3) A RX RSSI measurement should be possible without ever transmitting. However, in the particular RF chipset used in our implementation, the RSSI values are provided only after the radio has attempted to join the network. Therefore, during the start-up each face attempts to join the network which will cause its RSSI to be updated (including TX RSSI), which is then used in the algorithm. 


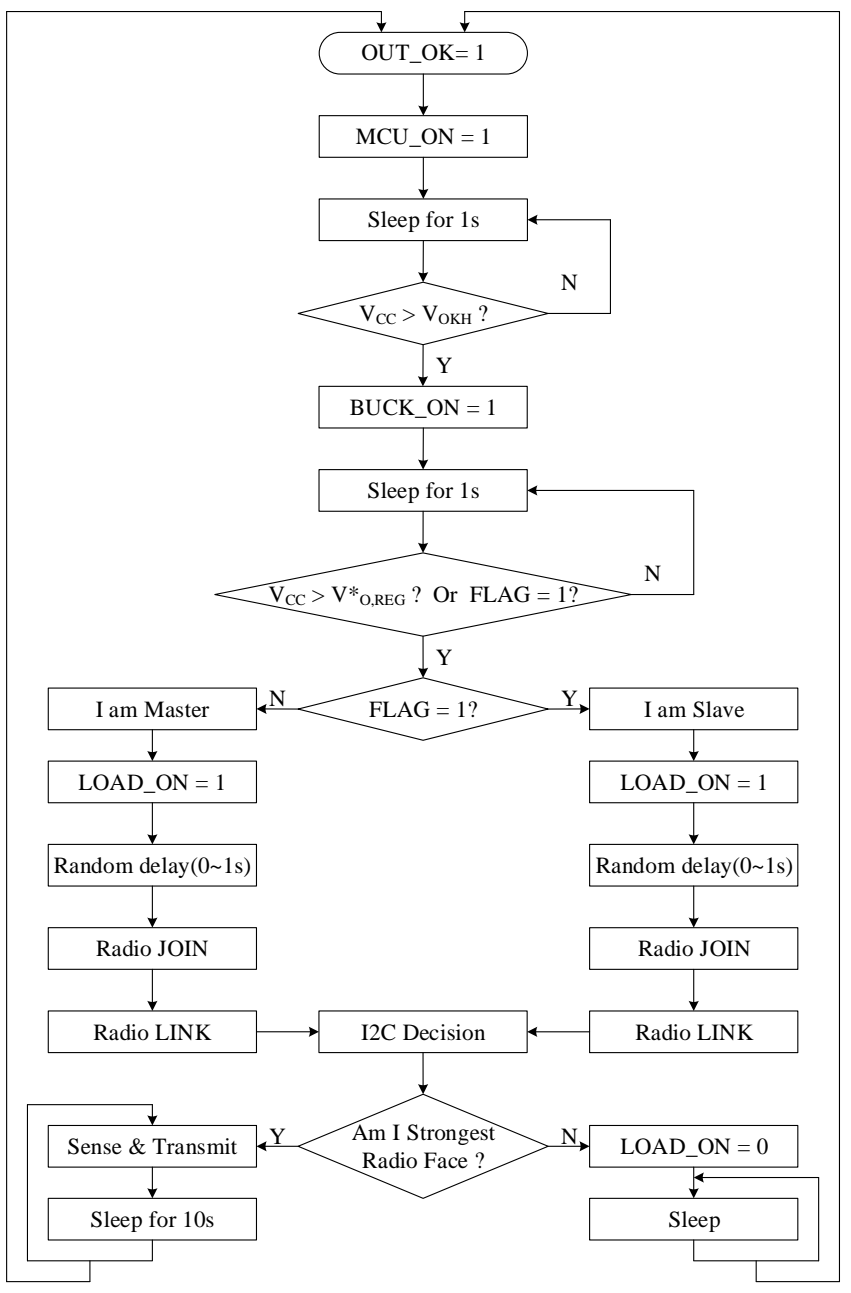

Fig. 7. Flow chart of the proposed algorithm.

\section{Design Aspects}

\section{A. Minimum Output Capacitance}

In steady-state the system sleeps for most of the time with a constant current consumption of a few micro-amperes. The major current consumption occurs only during the periodic radio transmission on the strongest radio face, which requires a few tens of milliamperes of pulse current. The pulse duration is very short, usually a few milliseconds. Also, each face undergoes a start-up sequence involving radio-JOIN and radioLINK operations, which require a similar amount of pulse current. The local output capacitor $C_{\mathrm{O}, \mathrm{UNREG}}$ must support all these transients, and is therefore calculated as

$$
C_{\mathrm{O}, \mathrm{UNREG}}=I_{\mathrm{O}, \mathrm{AV}} t_{\mathrm{PULSE}} /\left(V_{\mathrm{OKH}}-V_{\mathrm{OKL}}\right)
$$

where $t_{\mathrm{PULSE}}$ is the combined pulse duration for the three events - JOIN, LINK and transmit. Assuming no energy available from the boost converter (PMIC) side, $I_{\mathrm{O}, \mathrm{AV}}$ is the average current supplied by $C_{\mathrm{O}, \mathrm{UNREG}}$ during $t_{\mathrm{PULSE}}$. Note that $I_{\mathrm{O}, \mathrm{AV}}$ is not accurately predictable because of the different delay elements in the start-up sequence. Therefore, it is usually

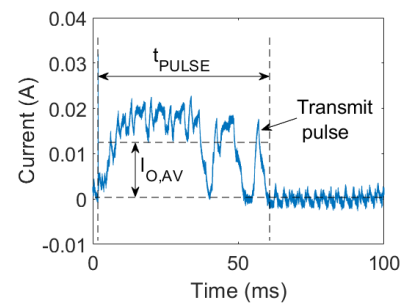

(a)

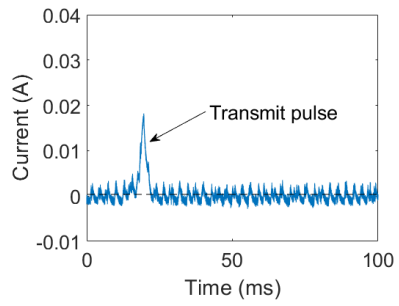

(b)
Fig. 8. An example of measurement of current consumption of the system (a) during start-up, and (b) during steady-state with periodic radio transmission. The measurements were taken in Keithley DMM6500 Digital Multimeter.

measured experimentally [7] using a constant voltage source with a nominal output voltage of

$$
V_{\mathrm{O}, \mathrm{NOM}}=\left(V_{\mathrm{OKH}}+V_{\mathrm{OKL}}\right) / 2 .
$$

One such measurement instance is shown in Fig. 8 for the example system described in the later sections.

Equation (5) merely indicates the minimum output capacitance needed. In practice, a higher value is used to support back-up operation when there is no energy available from the harvester. Ceramic capacitors are preferred in this study, because of their lower leakage current (X7R and $\mathrm{C} 0 \mathrm{G})$ as compared to tantalum capacitors. However, the effective value of capacitance of the ceramic capacitor drops drastically with the increase of dc bias [25], and this must be accounted for in the calculation of $C_{\mathrm{O}, \mathrm{UNREG}}$.

There is no such minimum requirement for the shared output capacitor $C_{\mathrm{O}, \mathrm{REG}}$. However, it is better to keep its value smaller than $C_{\mathrm{O} \text {,UNREG }}$ in order to keep the voltage build-up time of the shared bus short (time $t_{2}$ to $t_{3}$ in Fig. 6).

\section{B. Minimum Harvester Capacity}

Assuming the MCU is off, the average current needed for charging the capacitor $C_{\mathrm{O} \text {,UNREG }}$ from voltage $V_{\mathrm{OKL}}$ to $V_{\mathrm{OKH}}$ in $t_{\text {CHARGE }}$ time is obtained as

$$
I_{\text {CHARGE }}=C_{\mathrm{O}, \mathrm{UNREG}}\left(V_{\mathrm{OKH}}-V_{\mathrm{OKL}}\right) / t_{\mathrm{CHARGE}} \text {. }
$$

Ideally, $I_{\text {CHARGE }}$ is equal to the boost converter's (PMIC) average output current $I_{\mathrm{O}, \mathrm{BOOST}}$. But, in reality, $I_{\mathrm{O}, \mathrm{BOOST}}$ also includes the quiescent current of the PMIC and the leakage current of the output capacitors.

$$
I_{\mathrm{O}, \mathrm{BOOST}}=I_{\mathrm{CHARGE}}+I_{\mathrm{Q}}+I_{\mathrm{LEAK}}
$$

Therefore, for a known input voltage $V_{\mathrm{I}}$, the average input current required from the harvester can be found as

$$
I_{\mathrm{I}}=I_{\mathrm{O}, \mathrm{BOOST}} V_{\mathrm{O}, \mathrm{NOM}} /\left(\eta V_{\mathrm{I}}\right)
$$

where $\eta$ is the efficiency of the boost converter (PMIC). Equations (7), (8) and (9) determine the minimum average voltage and average current requirement from the harvester for a desired $t_{\text {CHARGE }}$ value. 
TABLE I

CURRENT CONSUMPTION

\begin{tabular}{ll}
\hline \hline Mode & Current \\
\hline MSP430F2274 MCU & \\
Active mode (AM) at 1 MHz, 3 V & $300 \mu \mathrm{A}$ \\
Low power mode: LPM0 & $55 \mu \mathrm{A}$ \\
Low power mode: LPM3 & $0.9 \mu \mathrm{A}$ \\
Low power mode: LPM4 & $0.1 \mu \mathrm{A}$ \\
\hline CC2500 radio & $18.8 \mathrm{~mA}$ \\
RX input signal at the sensitivity limit, 250 kbps & $21.2 \mathrm{~mA}$ \\
TX (0 dBm) & $0.4 \mu \mathrm{A}$ \\
Sleep mode & $0.5 \mu \mathrm{A}$ \\
\hline bq25570 PMIC quiescent current & $1.0 \mu \mathrm{A}$ \\
Leakage current of output capacitors per face (appr.) & $0.5 \mu \mathrm{A}$ \\
\hline Leakage/quiescent current of misc. components per face (appr.)
\end{tabular}

\section{VALIDATION}

\section{A. Example System}

An example system was implemented using bq25570 [15] as the PMIC, MSP430F2274 [23] as the MCU and CC2500 as the RF transceiver (radio), all from Texas Instruments. For the sensor we used the integrated temperature sensor of the MSP430F2274 MCU. The bq25570 PMIC has a boost converter for unregulated output (local) and a buck converter for regulated output (shared), both operated in Pulse Frequency Modulation (PFM) mode to maintain high efficiency at the extremely low power of the harvesters. The combination of MSP430 MCU, CC2500 transceiver, and the SimpliciTI RF network protocol [26] provides a low power WSN application framework. Table I shows the various current consumption of these components as per their respective datasheets, which will be used later in the discussions.

As per the discussion in the previous section, we have used $3 \mathrm{mF}$ capacitance (ceramic) for the local output port and a 1 $\mathrm{cm}^{2} \mathrm{PV}$ panel (KXOB22-04X3F from IXYS) as the harvester on each face of the cube. Also, a total of $1.5 \mathrm{mF}$ capacitance, distributed equally among all the six faces, is used for the shared output port.

The developed laboratory prototype of the proposed cube is shown in Fig. 9. As illustrated by the computer aided design (CAD) rendering of the system in Figs. 9(a) and (b), it is a modular design with each face identical to the others except for the connectors. Each face has four connectors on its four edges to connect to its four neighbours. The same footprint has been used for both male and female connectors, and the six faces are grouped in three pairs - two with all four male connectors, two with all four female connectors, and two with two male + two female connectors. Such design helps to keep the cube easy to assemble and dismantle as and when required, for example to replace a damaged face.

\section{B. Experimental Results and Discussions}

For easy understanding, the results of the experiments with three faces are presented here. The set-up is illustrated in Fig. 10. The energy harvester (EH) at the input of each face is emulated by a constant voltage source with appropriate series resistance to adjust the available input energy. An eZ430RF2500T dongle from Texas Instruments plugged in to a PC is used as the base station.
As depicted in Fig. 10, face1 has the highest available energy, face2 has some available energy lesser than that of face 1 , and face 3 has no available energy, at their respective input sides. However, face 3 has the best line-of-sight with the base station. The proposed algorithm has to wake up face3 using energy from the other two faces, find out that face 3 is the strongest radio, and then channel the harvested energy from all the other sides to keep only the face 3 radio active for periodic transmission.

The following waveforms have been captured in Keysight DSOX2024A oscilloscope using standard passive probes.

Fig. 11 shows the build-up of voltages on the three local output ports and the shared output port for the setup shown in Fig. 10. It is easy to see the similarity of the charging pattern with that illustrated in Fig. 6. A phenomenon called the end of cold-start period, which is specific to the bq25570 PMICs [15], can also be observed here.

Another extreme case where only face1 has available energy at its input is shown in Fig. 12. The charging pattern here is mostly similar to that in Fig. 11, except that it takes longer to reach the point of $\mathrm{I} 2 \mathrm{C}$ decision in this case. A zoomed look around the I2C decision point as shown in Fig. 12(a) reveals some important events: the turning on of MCU3 when $V_{\mathrm{O}, \mathrm{UNREG} 3}$ exceeds $3 \mathrm{~V}$, and the periodic radio transmission (every $10 \mathrm{~s}$ ) by radio3 after the I2C decision. Note that none of the other three voltages $\left(V_{\mathrm{O}, \mathrm{UNREG} 1}, V_{\mathrm{O}, \mathrm{UNREG} 2}\right.$, and $\left.V_{\mathrm{O}, \mathrm{REG}}\right)$ has this periodic undershoot, indicating that face 3 is the strongest radio face and therefore the only face performing the radio transmission.

Figs. 13 and 14 demonstrate the I2C decision making process to choose the strongest radio face. Since face 1 is the strongest energy face, MCU1 (bearing address 0x01) becomes the I2C master. It reads the RSSI values from the other two I2C slave faces, decides the strongest radio face (in this case face 3 ) based on the maximum RSSI value, and writes the address of the strongest radio face (0x03 here) to the other two I2C slave faces. Based on this decision, face 1 and face 2 turns off their LOAD_ON signals and go to sleep, while face 3 continues to transmit the sensed value periodically.

\section{Performance Comparison with a Reference System}

A simple energy performance comparison can be made between the proposed cube and a conventional WSN (reference system) implemented with only one PMIC, one MCU, and one radio using the program flow diagram as shown in Fig. 15 highlighting the current consumption at each state, along with its duration. It is noted that, in the steady-state, the proposed cube has five faces sleeping in LPM4 consuming an extra power of $5 \times 3 \mathrm{~V} \times 2.1 \mu \mathrm{A}=31.5 \mu \mathrm{W}$. Clearly, this is the only extra power the proposed cube needs in the steady-state as compared to the reference system.

An energy comparison, instead of power comparison, is more sensible for the start-up phase since it happens only once and the start-up durations for the two systems are different. For the reference system, the only states during start-up are - Initialize and JOIN \& LINK, which require a total of 635 $\mu \mathrm{J}$ of energy. In contrast, the proposed cube needs $4874 \mu \mathrm{J}$ of 


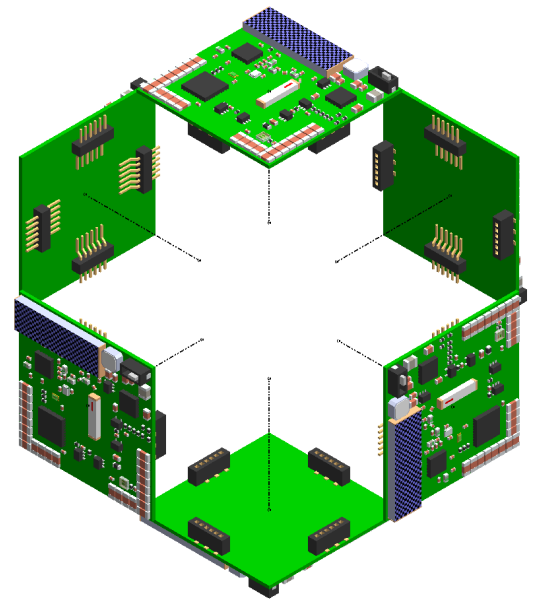

(a)

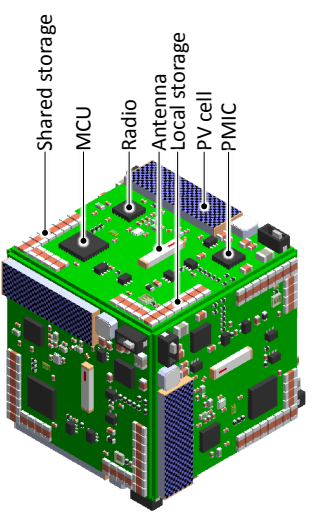

(b)

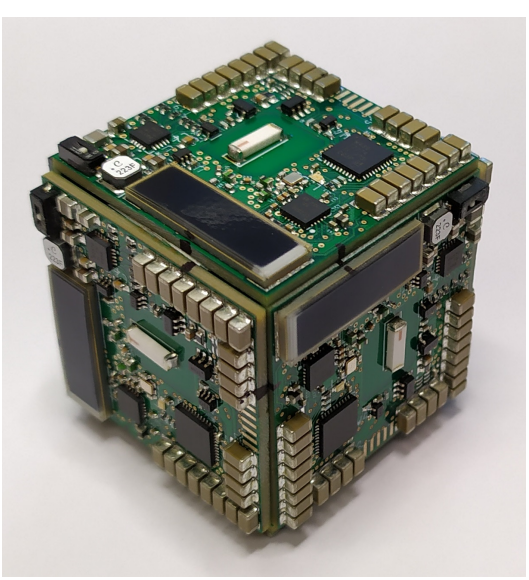

(c)

Fig. 9. Three dimensional CAD rendering of the proposed cube - (a) exploded view, (b) collapsed view, and (c) experimental prototype of the proposed cube. Dimension: $35 \mathrm{~mm}$ x $35 \mathrm{~mm}$ x $35 \mathrm{~mm}$.

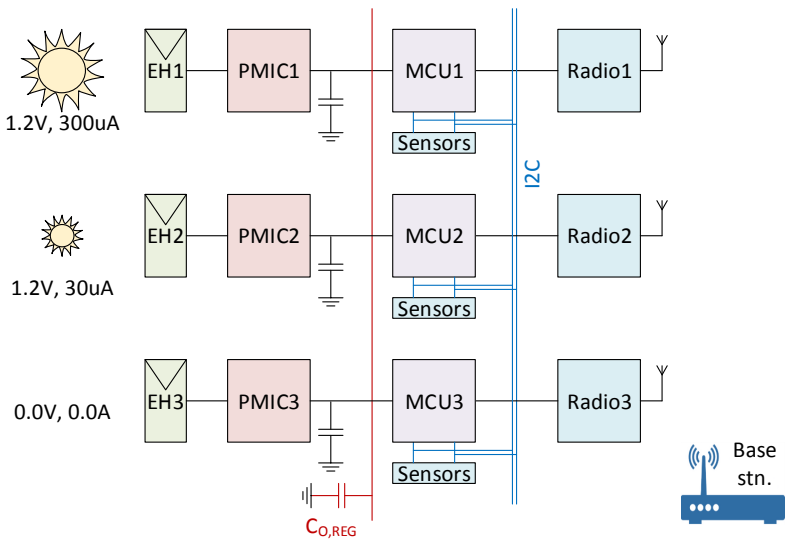

Fig. 10. Experimental setup for demonstration of the proposed algorithm using three faces of the cube.

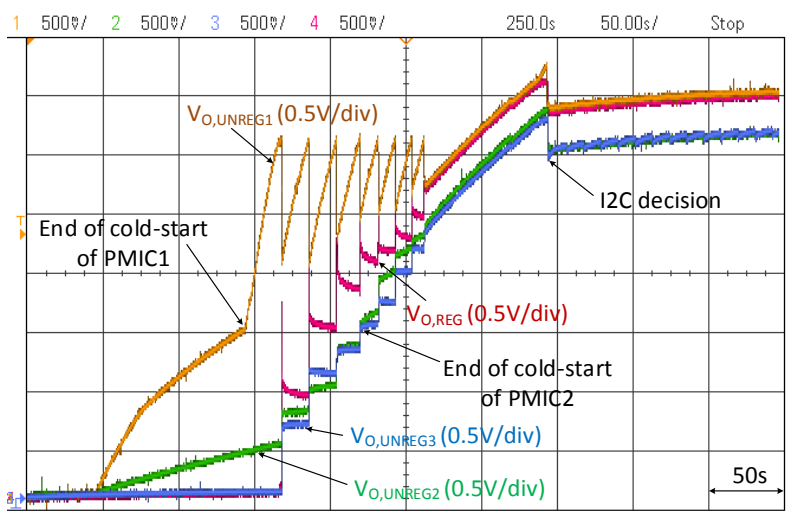

Fig. 11. Build-up of the local and the shared output voltages for the example case with three faces as shown in Fig. 10.

energy for its start-up phase. This large difference of energy is due to the fact that, unlike the steady-state, the start-up of the proposed cube involves all six faces undergoing the JOIN

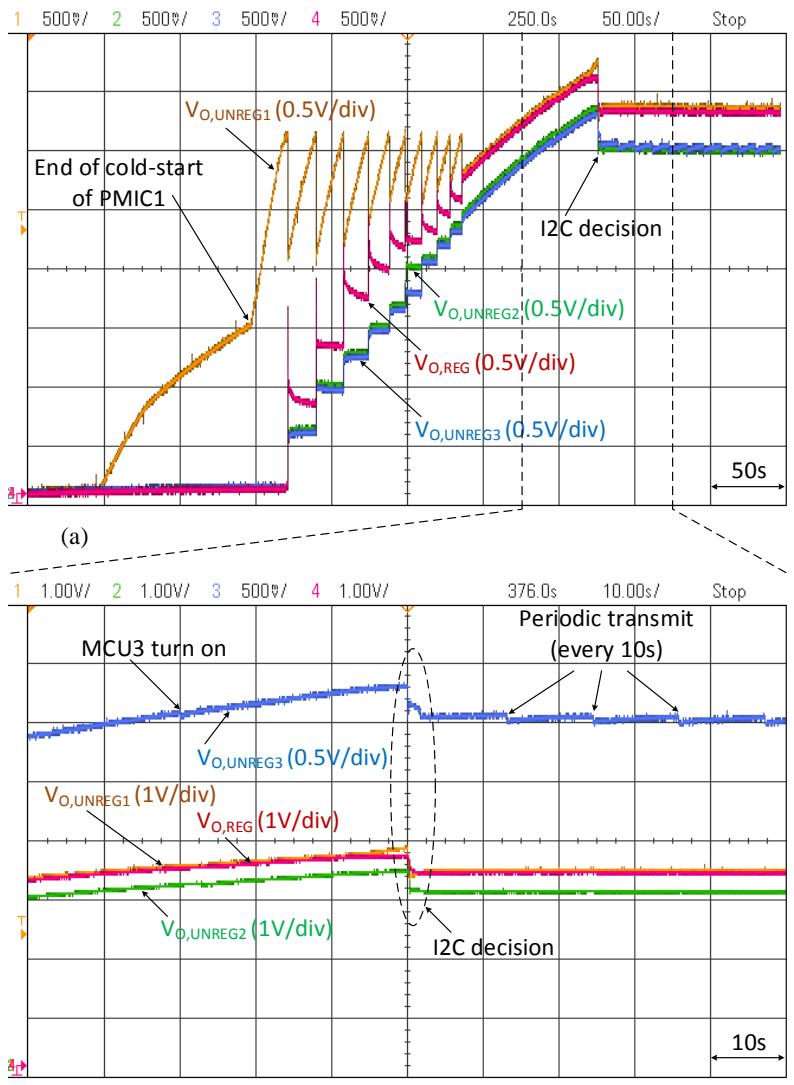

(b)

Fig. 12. (a) Building-up of the local and the shared output voltages when only face 1 has available energy. (b) Zoomed look showing turn-on of MCU3 and periodic radio transmission by radio3. Note the difference in voltage scale for $V_{\mathrm{O}, \mathrm{UNREG} 3}(0.5 \mathrm{~V} / \mathrm{div})$ w.r.t. the other voltages $(1 \mathrm{~V} /$ div $)$.

\& LINK state, which is the major energy consuming state.

As mentioned in section II, it is likely that the energy consumption of the proposed cube can be reduced to similar levels as the reference system by use of directional antennas, 


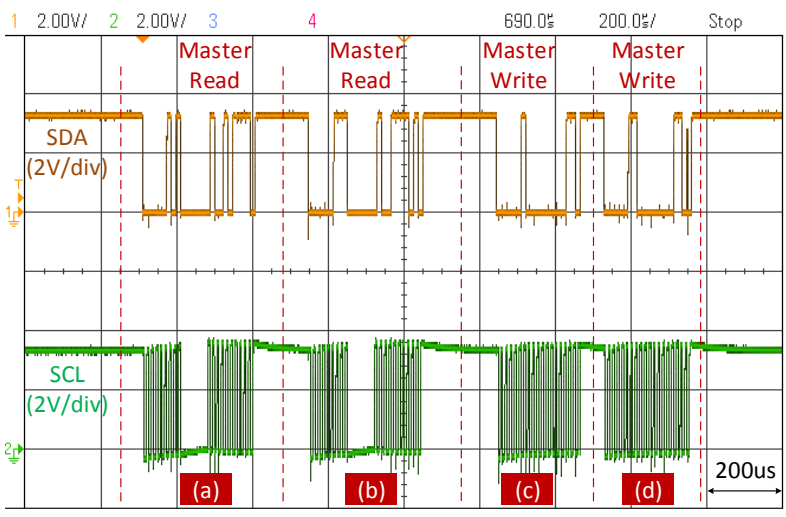

Fig. 13. Demonstration of the decision making process using $12 \mathrm{C}$ communication among the three faces.

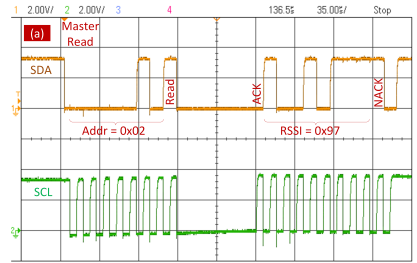

(a) Master read

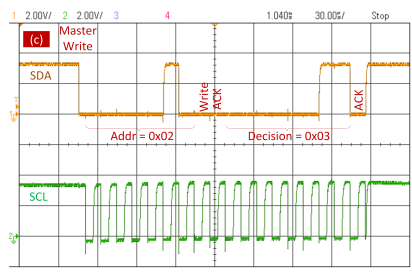

(c) Master write

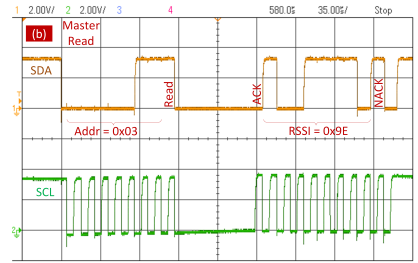

(b) Master read

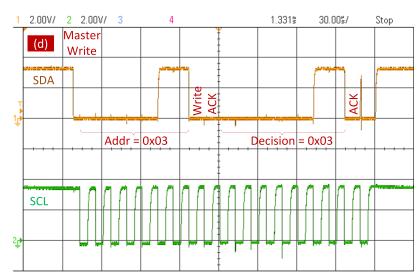

(d) Master write
Fig. 14. Zoomed look into Fig. 13. Note that MCU1 (bearing address $0 \times 01$ ) is the I2C master here. It reads the RSSI values from the other two I2C slave faces, decides the strongest radio face (face 3 in this case) based on the maximum RSSI value, and writes the address of the strongest radio face (0x03 here) to the other two I2C slave faces.

which is out of scope of this paper.

A qualitative comparison of the proposed concept with some existing works is presented in Table II.

\section{DIScussions}

In essence, the proposed system tolerates the directional natures of both harvestable energy and radio communication. Although the implementation in the paper is PV-based, which has directional energy input, the proposed system is applicable to those WSNs where energy sources are not directional, e.g. vibration-energy-harvesting-based WSNs (although many vibrational harvesters do have a preferred direction of motion). In such systems, a successful radio communication is still dependent on proper alignment of transmitter and base station, and forces like vibration may cause movement that changes this alignment over time.

It should be noted that the choice of the components, and physical shielding of the node are some of the practical aspects

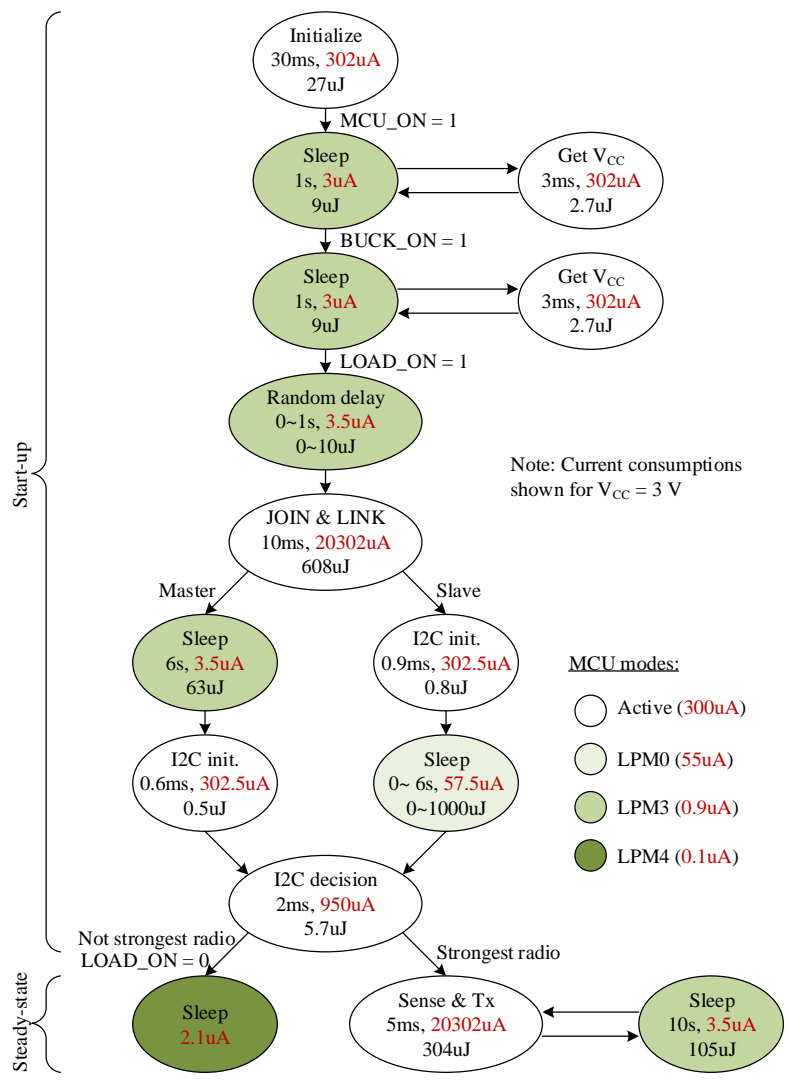

Fig. 15. Program flow of the example system showing duration of each state with its average current consumption.

that are application specific. For example, radiation shielding and use of rad-hard components would be necessary for use in nuclear radiation fields.

Lastly, a cube may not necessarily be the optimum threedimensional shape for the proposed device; we chose a cube primarily because it was easier to assemble in the laboratory using discrete printed circuit boards. Other regular shapes, for example a tetrahedron, may be more cost-effective depending on the particular application. The electrical configuration would be similar, with a only a change in the total number of faces.

\section{CONCLUSIONS}

A smart wireless sensor node is proposed in this paper to overcome the issues related to the placement and orientation of the sensor nodes in extreme and inaccessible environments. It has been shown that irrespective of how it is oriented in the target environment, the device ensures radio transmission to the base station as long as at least one of the faces is able to harvest energy, and at least one has the base station in its line-of-sight. Important design aspects have been covered to make the algorithm and the device as energy-efficient as possible using different depth of low-power modes available in the off-the-shelf components. 
TABLE II

COMPARISON WITH EXISTING WORKS

\begin{tabular}{lllll}
\hline \hline Work & Components & Active components in steady-state & MPPT of individual EH & Proper placement and orientation \\
\hline Conventional WSN & 1xEH, 1xPMIC, 1xMCU, 1xRF & All & Yes & Needed \\
Reference [2] & 2xEH,1xPMIC, 1xMCU, 1xRF & All & No & Needed \\
SolarDice [7] & 6xEH, 1xPMIC, 1xMCU, 1xRF & All & No & Needed \\
AmbiMax [27] & 3xEH,3xPMIC, 1xMCU, 1xRF & All & Yes & Needed \\
This work & 6xEH,6xPMIC, 6xMCU, 6xRF & 6xEH,6xPMIC, 1xMCU, 1xRF & Yes & Not Needed \\
\hline
\end{tabular}

\section{REFERENCES}

[1] V. C. Gungor and G. P. Hancke, "Industrial wireless sensor networks: Challenges, design principles, and technical approaches," IEEE Trans. Ind. Electron., vol. 56, no. 10, pp. 4258-4265, Oct. 2009.

[2] Y. K. Tan and S. K. Panda, "Energy harvesting from hybrid indoor ambient light and thermal energy sources for enhanced performance of wireless sensor nodes," IEEE Trans. Ind. Electron., vol. 58, no. 9, pp. 4424-4435, Sep. 2011.

[3] Z. J. Chew, T. Ruan, and M. Zhu, "Power management circuit for wireless sensor nodes powered by energy harvesting: On the synergy of harvester and load," IEEE Trans. Power Electron., vol. 34, no. 9, pp. 8671-8681, Sep. 2019.

[4] D. Newell and M. Duffy, "Review of power conversion and energy management for low-power, low-voltage energy harvesting powered wireless sensors," IEEE Trans. Power Electron., vol. 34, no. 10, pp. 9794-9805, Oct. 2019.

[5] S. Kim, R. Vyas, J. Bito, K. Niotaki, A. Collado, A. Georgiadis, and M. M. Tentzeris, "Ambient RF energy-harvesting technologies for selfsustainable standalone wireless sensor platforms," Proc. IEEE, vol. 102, no. 11, pp. 1649-1666, Nov. 2014.

[6] C. Yang and K. Chin, "On nodes placement in energy harvesting wireless sensor networks for coverage and connectivity," IEEE Trans. Ind. Informat., vol. 13, no. 1, pp. 27-36, Feb. 2017.

[7] "SolarDice (PMP9754): a sensor node in the internet of things (IoT) reference design," Texas Instruments, 2014.

[8] G. Werner-Allen, K. Lorincz, M. Ruiz, O. Marcillo, J. Johnson, J. Lees, and M. Welsh, "Deploying a wireless sensor network on an active volcano," IEEE Internet Comput., vol. 10, no. 2, pp. 18-25, Mar. 2006.

[9] D. Yang, S. Misra, X. Fang, G. Xue, and J. Zhang, "Two-tiered constrained relay node placement in wireless sensor networks: Computational complexity and efficient approximations," IEEE Trans. Mobile Comput., vol. 11, no. 8, pp. 1399-1411, Aug. 2012.

[10] S. Rani, S. H. Ahmed, R. Talwar, and J. Malhotra, "Can sensors collect big data? an energy-efficient big data gathering algorithm for a wsn," IEEE Trans. Ind. Informat., vol. 13, no. 4, pp. 1961-1968, Aug. 2017.

[11] A. Forster, Introduction to Wireless Sensor Networks. IEEE, 2016.

[12] O. Lopez-Lapena, M. T. Penella, and M. Gasulla, "A new mppt method for low-power solar energy harvesting," IEEE Trans. Ind. Electron., vol. 57, no. 9, pp. 3129-3138, Sep. 2010.

[13] F. I. Simjee and P. H. Chou, "Efficient charging of supercapacitors for extended lifetime of wireless sensor nodes," IEEE Trans. Power Electron., vol. 23, no. 3, pp. 1526-1536, May. 2008.

[14] "BQ25504: ultra low-power boost converter with battery management for energy harvester applications," Texas Instruments, Jun. 2015.

[15] "BQ25570: nano power boost charger and buck converter for energy harvester powered applications," Texas Instruments, Dec. 2018.

[16] "LTC3105: 400ma step-up dc/dc converter with maximum power point control and 250mv start-up," Linear Technology, 2010.

[17] "SPV1050: ultralow power energy harvester and battery charger," STMicroelectronics, May. 2018.

[18] “CC430 family user's guide," Texas Instruments, Jan. 2013.

[19] "LTC3108: ultralow voltage step-up converter and power manager," Linear Technology, 2010.

[20] W. Jung, S. Oh, S. Bang, Y. Lee, Z. Foo, G. Kim, Y. Zhang, D. Sylvester, and D. Blaauw, "An ultra-low power fully integrated energy harvester

[22] E. Dallago, A. L. Barnabei, A. Liberale, P. Malcovati, and G. Venchi, "An interface circuit for low-voltage low-current energy harvesting based on self-oscillating switched-capacitor voltage doubler," IEEE $J$. Solid-State Circuits, vol. 49, no. 12, pp. 2800-2811, Dec. 2014.

[21] X. Liu, K. Ravichandran, and E. Sanchez-Sinencio, "A switched capacitor energy harvester based on a single-cycle criterion for mppt to eliminate storage capacitor," IEEE Trans. Circuits Syst. I: Reg. Papers, vol. 65, no. 2, pp. 793-803, Feb. 2018.

systems," IEEE Trans. Power Electron., vol. 30, no. 3, pp. 1411-1420, Mar. 2015.

[23] “MSP430x2xx: family user's guide," Texas Instruments, Jul. 2013.

[24] B. Ivey, R. Hegde, and A. Bhat, "AN1267: extreme low-power (XLP) PIC microcontrollers: An introduction to microchip's low-power devices," Microchip Technology Inc., Jul. 2016.

[25] "Capacitance and dissipation factor measurement of chip multilayer ceramic capacitors," Murata Mfg. Co. Ltd., Oct. 2006.

[26] "SimpliciTI: developers notes, simple modular rf network," Texas Instruments, 2011.

[27] C. Park and P. H. Chou, "Ambimax: Autonomous energy harvesting platform for multi-supply wireless sensor nodes," in 2006 3rd Annual IEEE Communications Society on Sensor and Ad Hoc Communications and Networks, vol. 1, pp. 168-177, Sep. 2006.

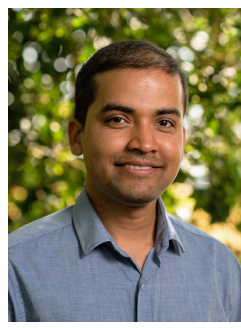

Kawsar Ali (S'14-M'19) received the B. Tech. degree from National Institute of Technology (NIT), Durgapur, India in 2011 and the PhD degree from National University of Singapore (NUS) in 2018, both in electrical engineering. From 2011 to 2014, he was with the Indian Oil Corporation Limited as an Operations Officer. $\mathrm{He}$ joined the Engineering Science Department of the University of Oxford in 2018 as a postdoctoral research associate, where he works on the power electronics design of ultra-low power energy harvesting systems, resonant power conversion, and application of wide-bandgap devices.

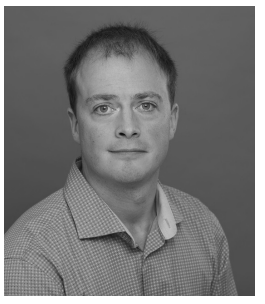

Daniel J. Rogers (M'09-SM'19) is an Associate Professor in the Department of Engineering Science at the University of Oxford, UK. He received the M.Eng. and Ph.D. degrees in Electrical and Electronic Engineering from Imperial College London, London, U.K., in 2007 and 2011 respectively. He conducts research in collaboration with industry and is an investigator on UK EPSRC research projects in the areas of power electronics, grid-scale energy storage and microgrids. Dan's research interests in power electronics range from active control of transistor switching, to circuit and control system design, through to novel applications enabled by wide-bandgap devices. 